\title{
Analysis and Control of the Singular System Model of Aphid Ecosystems
}

\author{
Jingna Liu, ${ }^{1,2}$ Tie Zhang $\left(\mathbb{D},{ }^{1}\right.$ Lichun Zhao, ${ }^{2}$ Bing Liu, ${ }^{2}$ and Xueying Wei ${ }^{3}$ \\ ${ }^{1}$ Department of Mathematics, Northeastern University, Shenyang 110006, China \\ ${ }^{2}$ School of Mathematics and Information Science, Anshan Normal University, Anshan 114007, China \\ ${ }^{3}$ Department of Mathematics, Liaoning Normal University, Dalian 116029, China \\ Correspondence should be addressed to Tie Zhang; tiezhang1956@163.com
}

Received 26 May 2017; Revised 14 November 2017; Accepted 28 December 2017; Published 19 February 2018

Academic Editor: Shoudong Huang

Copyright (C) 2018 Jingna Liu et al. This is an open access article distributed under the Creative Commons Attribution License, which permits unrestricted use, distribution, and reproduction in any medium, provided the original work is properly cited.

Considering the change of the parameter related to the natural enemy population and the impact on the aphid populations in the fold catastrophe manifold, the singular system model of aphid ecosystems is proposed. Combining singular system theory with catastrophe theory, the corresponding dynamics behaviors and the existence conditions of the impasse points are given by using the qualitative analysis. The biological significance of the analytical results is also discussed. The controllers are designed to make the aphid populations stabilize the refuge level by releasing natural enemy. Some numerical simulations are carried out to prove the results.

\section{Introduction}

Aphid populations are serious pests of wheat and many crops in the world. In some environment and physiography, aphid populations may lead to yield and economic losses [1-4]. Due to the fact that the fast reproduction of the aphid populations and the outbreak is always happening, insecticides are usually used to manage aphid pests. However, insecticides cause environment pollution and the lower quality of agricultural products. To avoid adverse effects of total reliance on insecticides and to ensure the well balanced ecosystems, there is a need to have ecofriendly management measures like releasing natural enemy [5]. Thus it is important to reveal the outbreak of aphid populations using mathematical models. In [6], the characteristics of catastrophe model are first used to describe and explain the phenomena of the population outbreak. In [7], a fold catastrophe model is proposed to explain the outbreak mechanism of the aphid populations by taking the proportion of winged aphid for the state variable and the effective leaf area for a control variable. In [8], on the basis of the Logistic model, a fold catastrophe model is built to show the complex dynamics behaviors of the aphid populations by taking the density of the aphid population for the state variable and the environmental factor for the main parameter.
In [9], based on the fold catastrophe model, a new model is proposed by applying the Allee effect to the logistic equation and the corresponding ecological interpretations are provided. According to the model above, Zhao et al. explain the sudden decrease of the aphid populations after spraying pesticides by means of real data and predict the outbreak of the aphid populations by determining catastrophe regions $[10,11]$. The results above show the application of catastrophe theory in aphid ecosystems. In fact, catastrophe theory is the theory related to the bifurcation theory, singularity theory, and structure stability [11].

Catastrophe is a widespread phenomenon in various fields, which frequently appears in engineering systems (such as the voltage load jump phenomenon in power systems). Some singular system models are usually used to describe the jump phenomenon in power systems and some results are obtained. Based on the singularity induced bifurcation of singular system, the complex dynamics behavior of power systems is studied and jump mechanisms are investigated [12-14]. According to the theory related to impasse points, the structure stability of power systems is analyzed and the impasse points in a circuit system are used to show the voltage load jump phenomenon $[15,16]$. 
What is the similarity between aphid populations' outbreak or sudden decrease and the voltage load jump phenomenon in power systems? Can the singular system models be used to describe the outbreak or sudden decrease phenomenon of aphid populations? It is what we are thinking about the focus of the paper.

The paper is organized as follows: In Section 2, the singular system model of aphid ecosystems is proposed. In Section 3, the dynamics behavior of aphid populations is discussed and the existence conditions of the impasse point are obtained by using qualitative analyses. In Section 4, the controllers are designed to keep aphid populations at refuge level and some numerical simulations are carried out to prove the results.

\section{Modelling}

Consider the following fold catastrophe manifold of the aphid populations [11]:

$$
0=r_{N} N(t)\left(1-\frac{N(t)}{k_{N}}\right)-\frac{m P N(t)}{N(t)+d}
$$

where $N(t)$ is the density of the aphid population; $P$ is the parameter related to the natural enemy population; $r_{N}, k_{N}$ represent the intrinsic rate and the environmental carrying capacity of the aphid population, respectively; $m, d$ represent the predation rate of the natural enemy population and the half saturation coefficient, respectively.

In model (1), $P$ is the parameter related to the natural enemy population. Without loss of generality, let it be the density of the natural enemy population and change as follows:

$$
\frac{d P(t)}{d t}=r_{P} P(t)\left(1-\frac{P(t)}{c N(t)+k_{p^{\prime}}}\right)
$$

where $r_{P}$ is the intrinsic rate of the natural enemy population; $c N(t)$ is the carrying capacity of the natural enemy population related to the density of the aphid population; and $k_{p^{\prime}}$ is the carrying capacity of the natural enemy population related to other environmental factor, such as crop and prey (except aphid) populations and so on, and it is the maximum density of the natural enemy population when the aphid population is absented.

Coupling the algebraic equation (1) with the differential equation (2), the singular system model for the aphid ecosystem is got:

$$
\begin{aligned}
\frac{d P(t)}{d t} & =r_{P} P(t)\left(1-\frac{P(t)}{c N(t)+k_{p^{\prime}}}\right), \\
0 & =r_{N} N(t)\left(1-\frac{N(t)}{k_{N}}\right)-\frac{m P(t) N(t)}{N(t)+d},
\end{aligned}
$$

For model (3), let

$$
\begin{aligned}
& x=h P, \\
& y=g N, \\
& \tau=q t ;
\end{aligned}
$$

applying transformation (4) to model (3), the following model is got:

$$
\begin{aligned}
\frac{d x(\tau)}{d \tau} & =x(\tau)\left(\frac{r_{N}}{q}-\frac{\left(g r_{P} / c q h\right) x(\tau)}{y(\tau)+k_{p^{\prime}} g / c}\right), \\
0 & =y(\tau)\left(\frac{r_{N}}{g}-\frac{r_{N}}{g^{2} k_{N}} y(\tau)-\frac{(m / h) x(\tau)}{y(\tau)+g d}\right) .
\end{aligned}
$$

Let

$$
\begin{aligned}
\frac{r_{N}}{g^{2} k_{N}} & =1, \\
\frac{m}{h} & =1, \\
\frac{g r_{P}}{c q h} & =1 ;
\end{aligned}
$$

the following are given:

$$
\begin{aligned}
& g=\sqrt{\frac{r_{N}}{k_{N}}}>0, \\
& h=m>0, \\
& q=\frac{g r_{P}}{c h}>0 .
\end{aligned}
$$

In model (5), let

$$
\begin{aligned}
& a=\frac{r_{P}}{g}, \\
& b=\frac{k_{P^{\prime}} g}{c}, \\
& l=\frac{r_{N}}{g}, \\
& n=g d .
\end{aligned}
$$

It becomes

$$
\begin{aligned}
\frac{d x(\tau)}{d \tau} & =x(\tau)\left(a-\frac{x(\tau)}{y(\tau)+b}\right), \\
0 & =y\left(l-y(\tau)-\frac{x(\tau)}{y(\tau)+n}\right) .
\end{aligned}
$$

Model (9) is discussed on the domain $D$, where $D$ is

$$
D=\{(x(\tau), y(\tau)) \mid x(\tau) \geq 0, y(\tau) \geq 0\} .
$$




\section{Qualitative Analysis}

3.1. Impasse Points. Consider the following model:

$$
\begin{aligned}
\frac{d x(t)}{d t} & =f(x(t), y(t)), \\
0 & =g(x(t), y(t)) .
\end{aligned}
$$

According to literature [17], the equilibria and singular points of model (11) are defined as follows:

$$
\begin{aligned}
& \mathrm{EQ} \triangleq\left\{\left(x_{0}, y_{0}\right) \mid f\left(x_{0}, y_{0}\right)=0, g\left(x_{0}, y_{0}\right)=0\right\} \\
& S \triangleq\left\{\left(x_{0}, y_{0}\right) \mid g\left(x_{0}, y_{0}\right)=0, \operatorname{det} \frac{\partial g\left(x_{0}, y_{0}\right)}{\partial y}=0\right\} .
\end{aligned}
$$

Lemma 1. For model (9),

(1) there may exist five equilibria $M_{1}(0,0), M_{2}(0, l)$, $M_{3}(a b, 0), M_{1}^{*}\left(x_{1}^{*}, y_{1}^{*}\right)$, and $M_{2}^{*}\left(x_{2}^{*} y_{2}^{*}\right)$, where $y_{1}^{*}<$ $y_{2}^{*}$ and $x_{i}^{*}, y_{i}^{*}, i=1,2$ satisfy the following equation set:

$$
\begin{aligned}
& y(\tau)=\frac{x(\tau)}{a}-b, \\
& x(\tau)=(l-y(\tau))(y(\tau)+n) ;
\end{aligned}
$$

(2) there exist two singular points $S_{1}\left(x_{S_{1}}, y_{S_{1}}\right)$ and $S_{2}\left(x_{S_{2}}\right.$, $\left.y_{S_{2}}\right)$, where $x_{S_{1}}=(l+n)^{2} / 4, y_{S_{1}}=(l-n) / 2, x_{S_{2}}=l n$, and $y_{S_{2}}=0$.

According to literature [16], let $\Psi=\left\{y \mid h\left(y_{0}, \lambda\right)=g\left(x_{0}+\right.\right.$ $\left.\left.\lambda f\left(x_{0}, y_{0}\right), y\right)=0\right\}$ be the induced solution curve, then the limit points and impasse points are defined as follows.

Definition 2. For model (11), for any $\lambda^{+}>\lambda_{0}$ (resp., $\lambda^{-}<\lambda_{0}$ ), there exists a neighbourhood $N_{0}$ of $\left(y_{0}, \lambda_{0}\right)$ such that $\Psi^{+} \cap$ $N=\varnothing$ (resp., $\left.\Psi^{-} \cap N=\varnothing\right)$, then the point $\left(y_{0}, \lambda_{0}\right)$ is called right (resp., left) limit point of the induced solution curve $\Psi$ at $\lambda=\lambda_{0}$, where $N$ is any of subsets of $N_{0}$ and

$$
\begin{aligned}
& \Psi^{+}=\left\{(y, \lambda) \mid h(y, \lambda)=0, \lambda_{0}<\lambda<\lambda^{+}\right\}, \\
& \Psi^{-}=\left\{(y, \lambda) \mid h(y, \lambda)=0, \lambda^{-}<\lambda<\lambda_{0}\right\} .
\end{aligned}
$$

Definition 3. For model (11), the point $Q\left(x_{0}, y_{0}\right)$ is forward (resp., backward) point, if $\left(y_{0}, 0\right)$ is a right (resp., left) limit point of the induced solution curve $\Psi$.

According to the definitions above, in order to find the impasse points, the following conditions are given:

(1) The impasse points must be the singular points.

(2) The corresponding limit point must be the solution to $h(y, \lambda)=0$.

(3) The corresponding limit point must be the solution to $\partial h(y, \lambda) / \partial y=0$.

(4) The linear coefficient for $\lambda$ must not be zero in the Taylor expansion of the $h(y, \lambda)$ at the point $\left(y_{S_{1}}, 0\right)$.
In fact, for singular points of model (9),

$$
\begin{aligned}
& \left.h(y, \lambda)\right|_{\left(y_{S_{1}}, 0\right)}=y_{S_{1}}\left(l-y_{S_{1}}-\frac{x_{S_{1}}}{y_{S_{1}}+n}\right)=0, \\
& \left.h(y, \lambda)\right|_{\left(y_{S_{2}}, 0\right)}=y_{S_{2}}\left(l-y_{S_{2}}-\frac{x_{S_{2}}}{y_{S_{2}}+n}\right)=0 ;
\end{aligned}
$$

thus, $\left(y_{S_{1}}, 0\right)$ and $\left(y_{S_{2}}, 0\right)$ are the solution to $h(y, \lambda)=0$.

Since

$$
\begin{aligned}
\left.\frac{\partial h(y, \lambda)}{\partial y}\right|_{\left(y_{S_{1}}, 0\right)}= & l-y_{S_{1}}-\frac{x_{S_{1}}}{y_{S_{1}}+n} \\
& +y_{S_{1}}\left(-1+\frac{x_{S_{1}}}{\left(y_{S_{1}}+n\right)^{2}}\right)=0,
\end{aligned}
$$

one has

$$
\begin{aligned}
\left.\frac{\partial h(y, \lambda)}{\partial y}\right|_{\left(y_{S_{2}}, 0\right)}= & l-y_{S_{2}}-\frac{x_{S_{2}}}{y_{S_{2}}+n} \\
& +y_{S_{2}}\left(-1+\frac{x_{S_{2}}}{\left(y_{S_{2}}+n\right)^{2}}\right) \\
= & l+\frac{(l+n)^{2}}{4 n} \neq 0 ;
\end{aligned}
$$

thus, $\left(y_{S_{1}}, 0\right)$ is the solution to $\partial h(y, \lambda) / \partial y=0$, but $\left(y_{S_{2}}, 0\right)$ is not.

And the Taylor expansion of the $h(y, \lambda)$ at the point $\left(y_{S_{1}}, 0\right)$ is

$$
\begin{aligned}
h(y, \lambda)= & h\left(y_{S_{1}}, 0\right)+\left.\frac{\partial h(y, \lambda)}{\partial \lambda}\right|_{\left(y_{S_{1}}, 0\right)}(\lambda-0) \\
& +\left.\frac{\partial h(y, \lambda)}{\partial y}\right|_{\left(y_{S_{1}}, 0\right)}\left(y-y_{S_{1}}\right) \\
& +\left.\frac{\partial^{2} h(y, \lambda)}{\partial \lambda^{2}}\right|_{\left(y_{S_{1}}, 0\right)}(\lambda-0)^{2} \\
& +\left.\frac{\partial^{2} h(y, \lambda)}{\partial y^{2}}\right|_{\left(y_{S_{1}}, 0\right)}\left(y-y_{S_{1}}\right)^{2}+o\left(\varepsilon^{3}\right) \\
= & -\frac{l^{2}-n^{2}}{4}\left(a-\frac{(l+n)^{2}}{2(l-n+2 b)}\right) \lambda \\
& -\frac{l-n}{l+n}\left(y-\frac{l-n}{2}\right)+o\left(\varepsilon^{3}\right)
\end{aligned}
$$

thus, the following result is got.

Theorem 4. For model (9),

(1) if $a<(l+n)^{2} / 2(l-n+2 b)$, then the point $\left(y_{S_{1}}, 0\right)$ is a left limit point of the induced solution curve $\Psi$, and the point $S_{1}$ is a backward impasse point; 


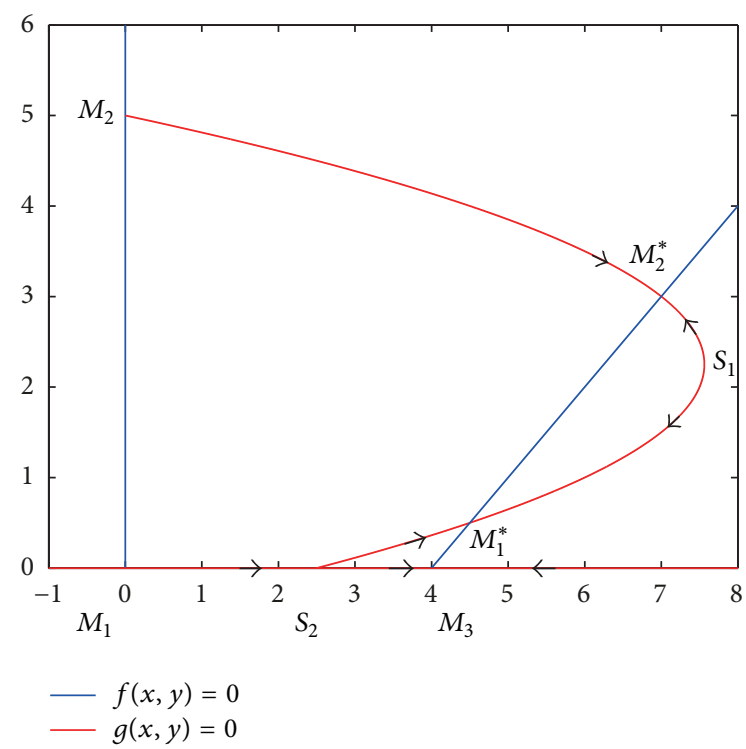

FIGURE $1: l>n, a b>\ln$ and $a=1$.

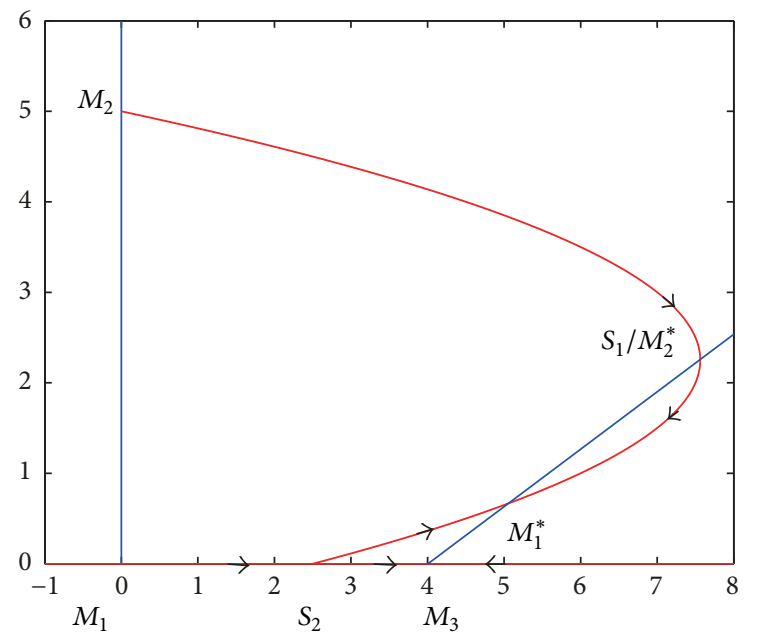

$f(x, y)=0$
$-g(x, y)=0$

Figure 2: $l>n, a b>\ln$ and $a=1.2$.

(2) if $a>(l+n)^{2} / 2(l-n+2 b)$, then the point $\left(y_{S_{1}}, 0\right)$ is a right limit point of the induced solution curve $\Psi$, and the point $S_{1}$ is a forward impasse point;

(3) if $a=(l+n)^{2} / 2(l-n+2 b)$, then there is no limit point or impasse point.

3.2. Qualitative Analysis Results. Based on the characteristics of the equilibria, singular points, and impasse points, using the geometrical analysis method, the qualitative analysis results of the singular system model (9) are obtained.

Theorem 5. The equilibria $M_{1}, M_{2}$ are both unstable nodes, and the equilibrium $M_{3}$ is a stable node.

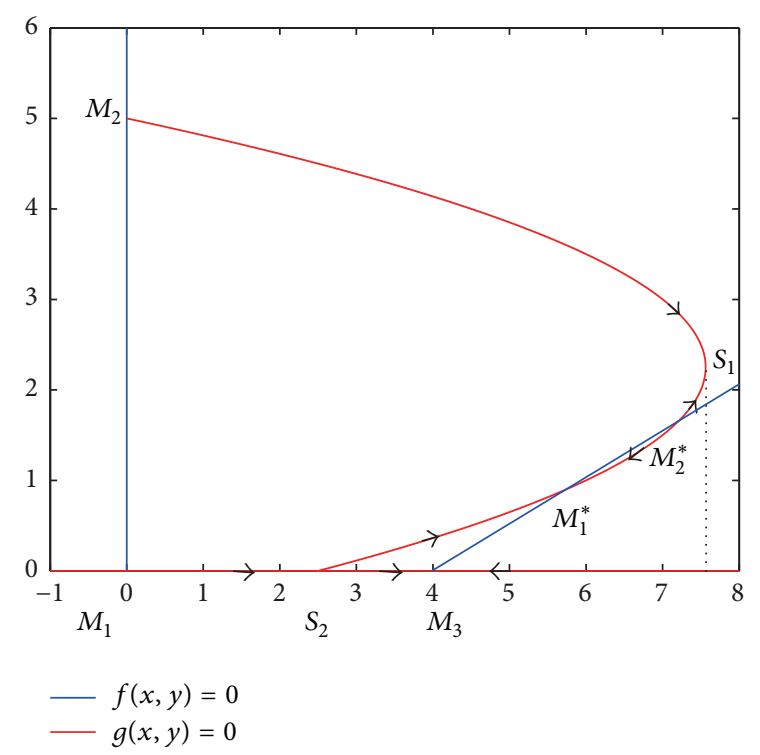

FiguRE 3: $l>n, a b>\ln$ and $a=1.5$.

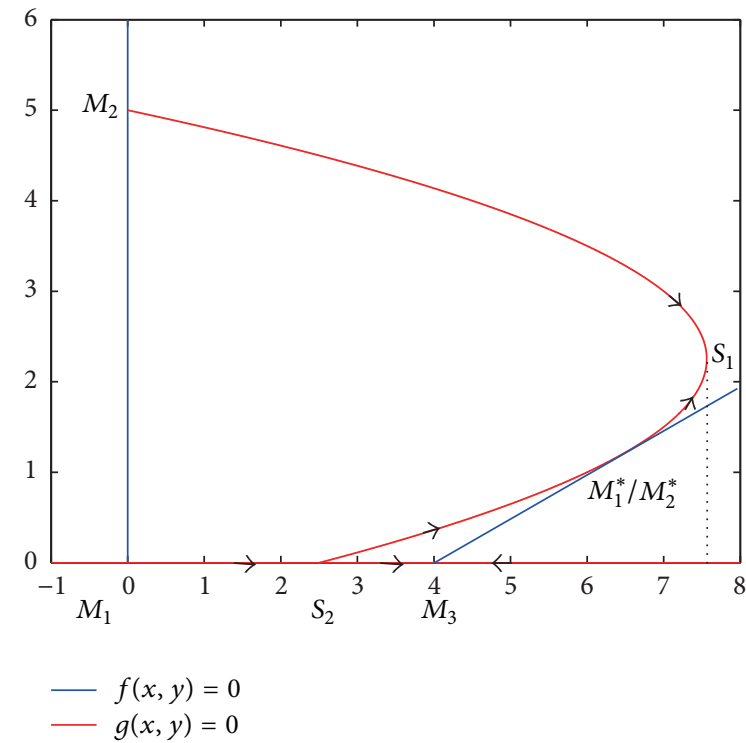

Figure $4: l>n, a b>\ln$ and $a=2$.

Theorem 6. For model (9), ifl $>n$ and $a b>\ln$, the qualitative analysis results are as follows:

(1) If the points $M_{1}^{*}$ and $M_{2}^{*}$ are on either side of point $S_{1}$, respectively, then they are both stable nodes and the point $S_{1}$ is a backward impasse point (see Figure 1).

(2) If the points $M_{2}^{*}$ and $S_{1}$ coincide, then that is a saddle point; the point $M_{1}^{*}$ is a stable node; and there is no impasse point (see Figure 2).

(3) If the points $M_{1}^{*}$ and $M_{2}^{*}$ locate below the point $S_{1}$, then the point $M_{1}^{*}$ is a stable node; the point $M_{2}^{*}$ is a saddle point; and the point $S_{1}$ is a forward impasse point (see Figure 3).

(4) If the points $M_{1}^{*}$ and $M_{2}^{*}$ coincide, then that is a saddle point and the point $S_{1}$ is a forward impasse point (see Figure 4). 


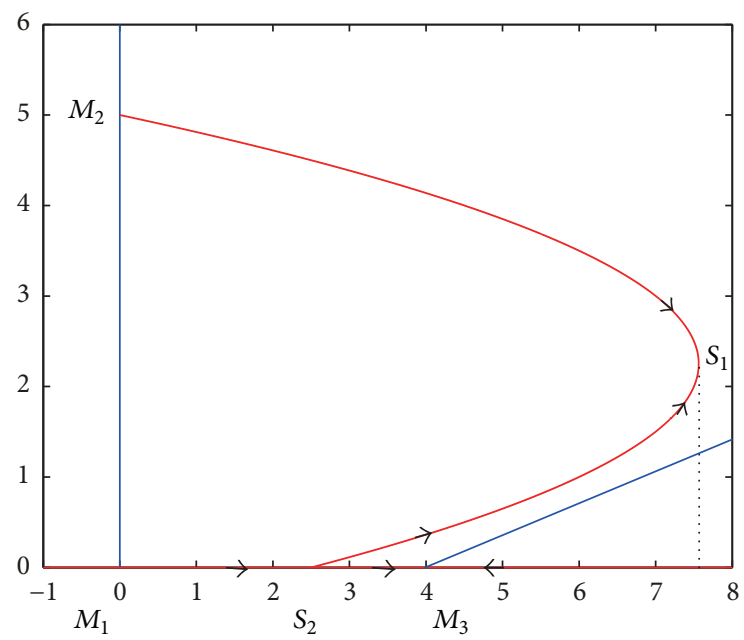

$f(x, y)=0$
$-g(x, y)=0$

Figure 5: $l>n, a b>\ln$ and $a=3$.

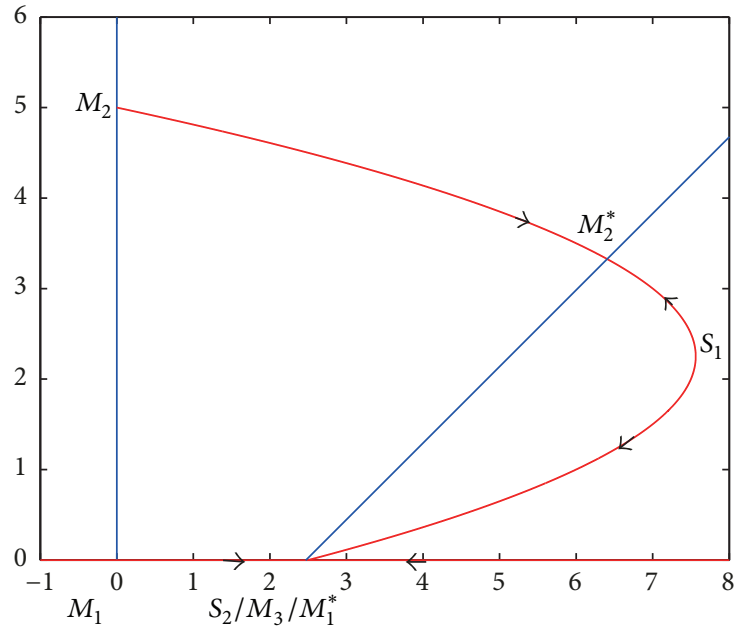

$\begin{aligned} f(x, y) & =0 \\ -g(x, y) & =0\end{aligned}$

Figure $6: l>n, a b=\ln$ and $a=1.25$.

(5) If the points $M_{1}^{*}$ and $M_{2}^{*}$ disappear, then the point $S_{1}$ is a forward impasse point (see Figure 5).

Theorem 7. For model (9), if $l>n$ and $a b=\ln$, the points $M_{1}^{*}$, $M_{1}$, and $S_{2}$ and the qualitative analysis results are as follows:

(1) If the point $M_{2}^{*}$ locates above the point $S_{1}$, then it is a stable node; the point $M_{1}^{*}$ is a stable node; and the point $S_{1}$ is a backward impasse point (see Figure 6).

(2) If the points $M_{2}^{*}$ and $S_{1}$ coincide, then that is a saddle point; the point $M_{1}^{*}$ is a stable node; and there is no impasse point for this case (see Figure 7).

(3) If the point $M_{2}^{*}$ locate below the point $S_{1}$, then it is a unstable node; the point $M_{1}^{*}$ is a stable node; the point $M_{2}^{*}$ is a

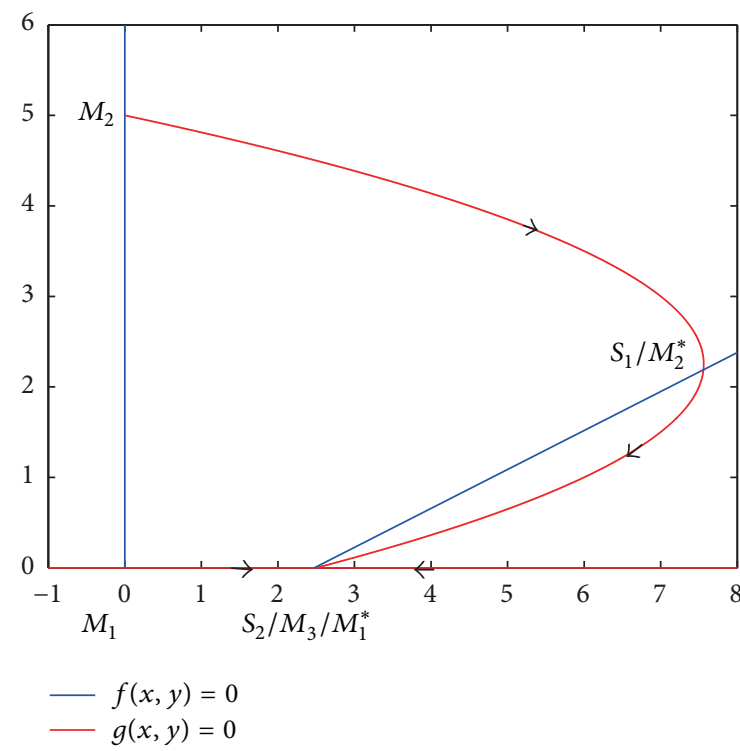

Figure 7: $l>n, a b=\ln$ and $a=2.2$.

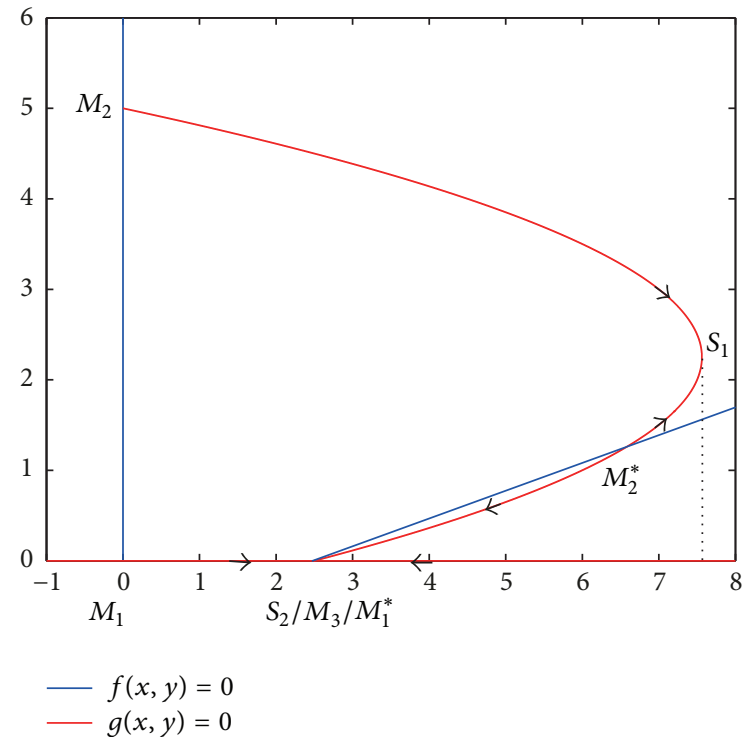

Figure 8: $l>n, a b=\ln$ and $a=3.5$.

saddle point; and the point $S_{1}$ is a forward impasse point (see Figure 8).

(4) If the points $M_{2}^{*}$ disappear, then the point $M_{1}^{*}$ is a saddle point and the point $S_{1}$ is a forward impasse point (see Figure 9).

Theorem 8. For model (9), if $l>n$ and $a b<\ln$, the point $M_{1}^{*}$ disappears and the qualitative analysis results are as follows:

(1) If the point $M_{2}^{*}$ locates above the point $S_{1}$, then it is a stable node and the point $S_{1}$ is a backward impasse point (see Figure 10).

(2) If the points $M_{2}^{*}$ and $S_{1}$ coincide, then that is a saddle point and there is no impasse point for this case (see Figure 11). 


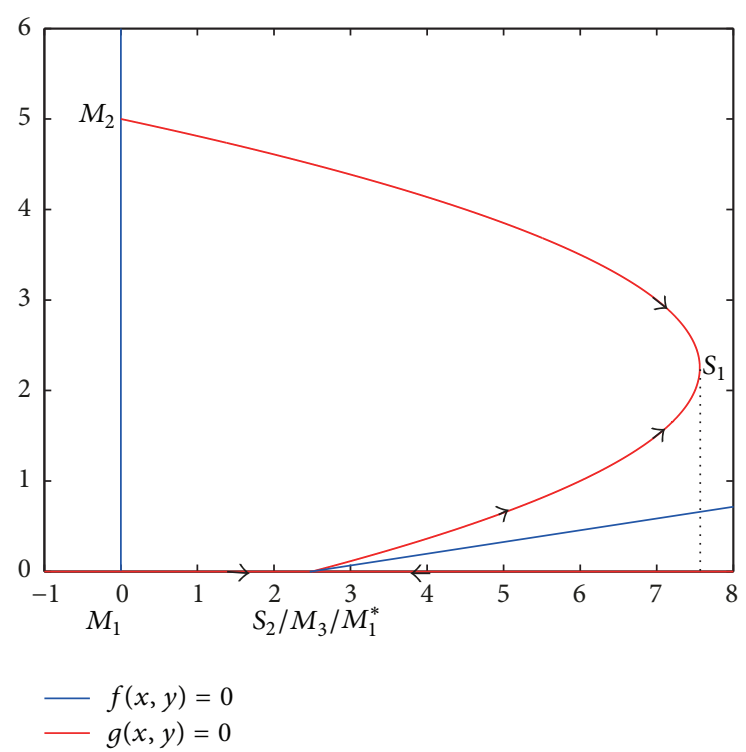

FIGURE 9: $l>n, a b=\ln$ and $a=5.5$.

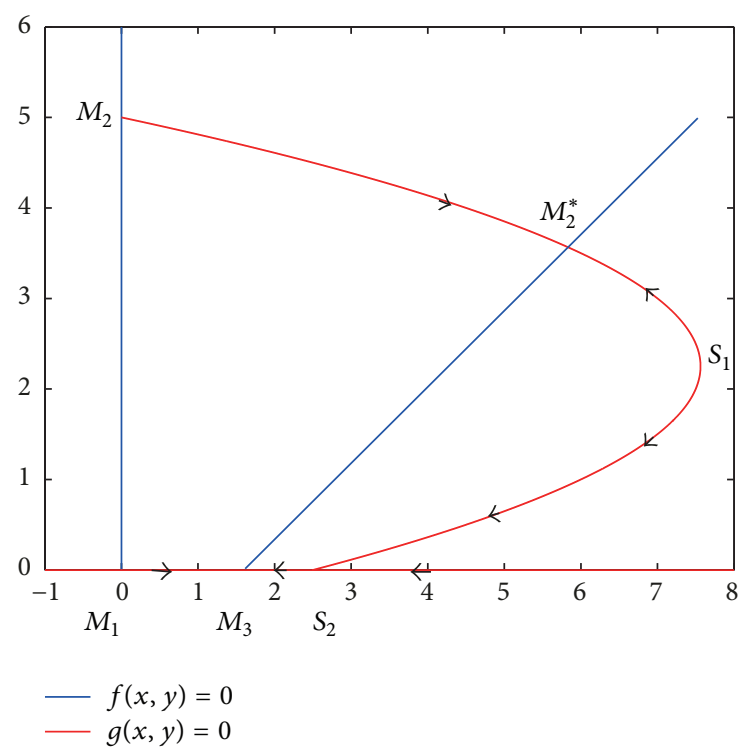

FIgURE 10: $l>n, a b<\ln$ and $a=1.5$.

(3) If the point $M_{2}^{*}$ locate below the point $S_{1}$, then it is a unstable node and the point $S_{1}$ is a forward impasse point (see Figure 12).

Theorem 9. For model (9), if $l \leq n$, the points $M_{1}^{*}, S_{1}$ and $S_{2}$ disappear and the qualitative analysis results are as follows:

(1) If $a b<\ln$, then the point $M_{2}^{*}$ disappears, too (see Figure 13).

(2) If $a b=\ln$, then the points $M_{2}^{*}$ and $M_{3}$ coincide and that is a stable node (see Figure 14).

(3) If $a b>\ln$, then the point $M_{2}^{*}$ is a stable node (see Figure 15).

Remark 10. For model (9), if the point $S_{1}$ is the forward impasse point, then there is catastrophe, that is, the aphid

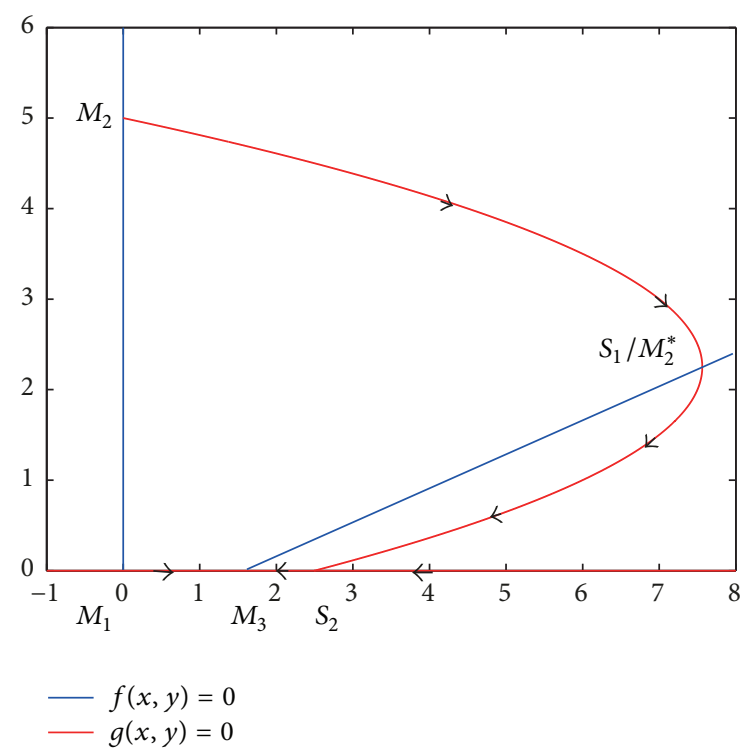

FIGURE 11: $l>n, a b<\ln$, and $a=2.5$.

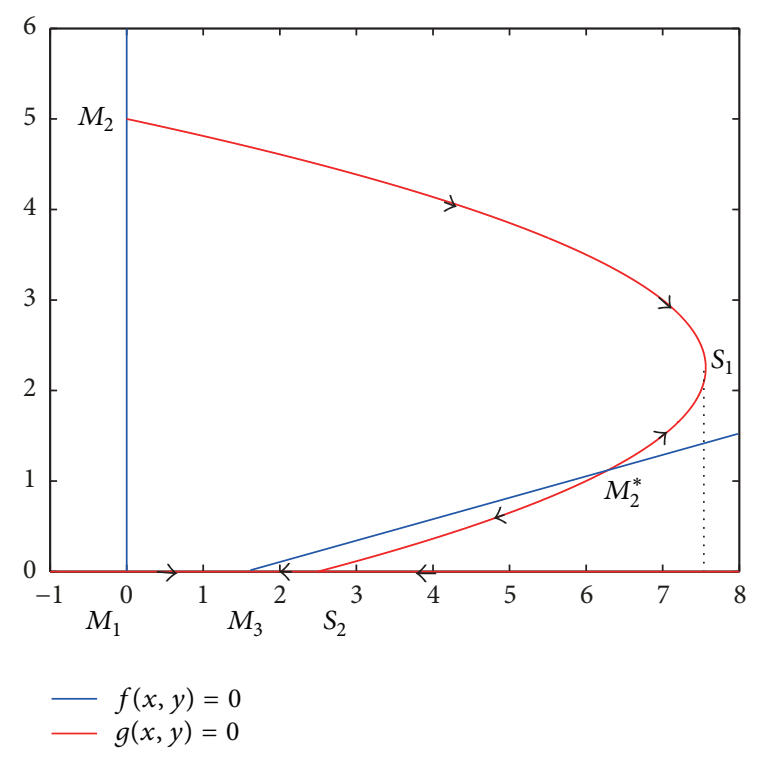

FIGURE 12: $l>n, a b<\ln$, and $a=3$.

population outbreak or sudden decrease (see Figures 3, 4, 5, 8,9 , and 12).

3.3. Biological Significance. According to the qualitative analysis and the corresponding figures, $a$ is an important parameter, which has a positive correlation with the intrinsic rate of the natural enemy population and the environmental carrying capacity of the aphid population and negative correlation with the intrinsic rate of the aphid population. The stable state of the aphid ecosystems will change over the increase of the parameter $a$. Moreover, for different initial states, the corresponding change process will be different.

In fact, all the qualitative analysis results can be divided into two categories: the first one is that the lower part of the 


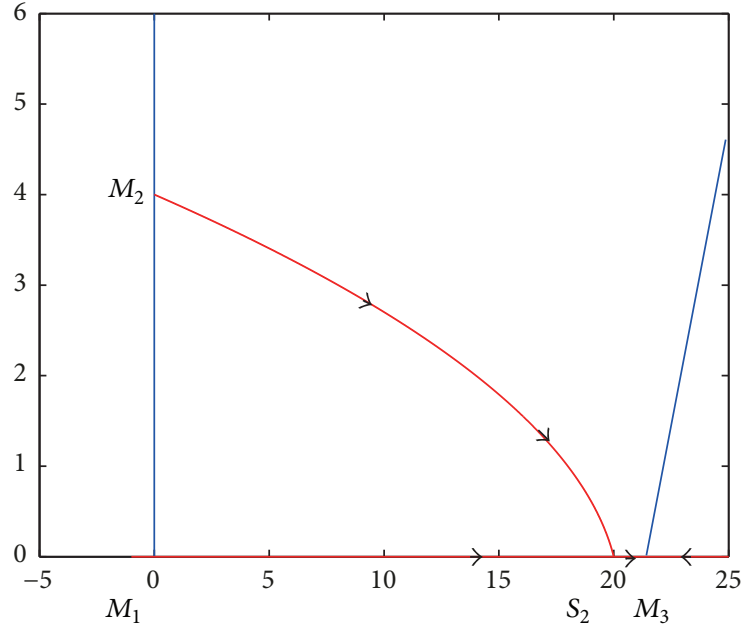

$-f(x, y)=0$

$-g(x, y)=0$

FIGURE 13: $l \leq n, a b>\ln$, and $a=1.5$.

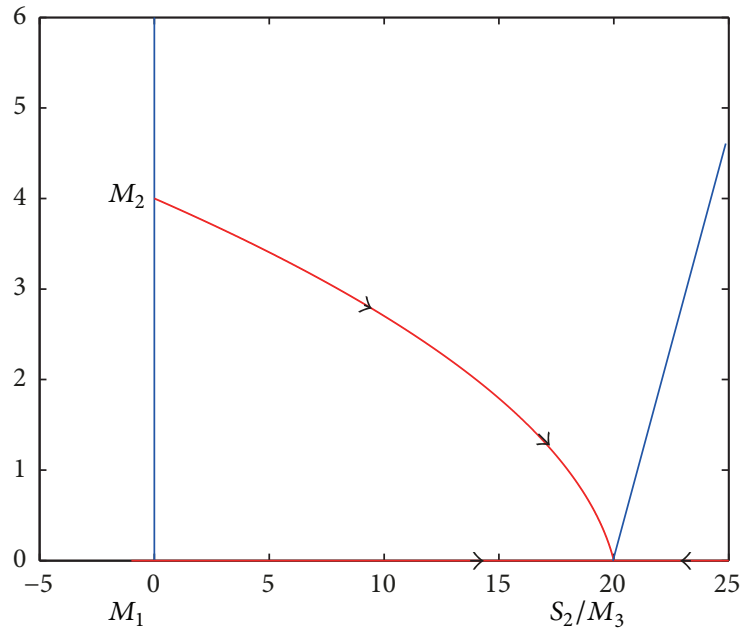

$f(x, y)=0$
$-g(x, y)=0$

FIGURE 14: $l \leq n, a b=\ln$, and $a=2.5$.

parabola $x(\tau)=(l-y(\tau))(y(\tau)+n)$ intersects with the $x$ axis and the corresponding results are Theorems $6-8$. The second one is that the upper part of the parabola intersects with the $x$-axis and the corresponding result is Theorem 9. And the following biological significance is discussed to the two categories.

For the first category, one has the following:

(1) If the environmental carrying capacity of the natural enemy population is larger than $x_{S_{2}}$, the following biological significance is got: when the density of the aphid population of the initial state is higher than $y_{S_{1}}$, with gradual increase of the parameter $a$, the system state is changed from the final state with the high aphid population density to the occurrence of the beneficial catastrophe; that is, the aphid

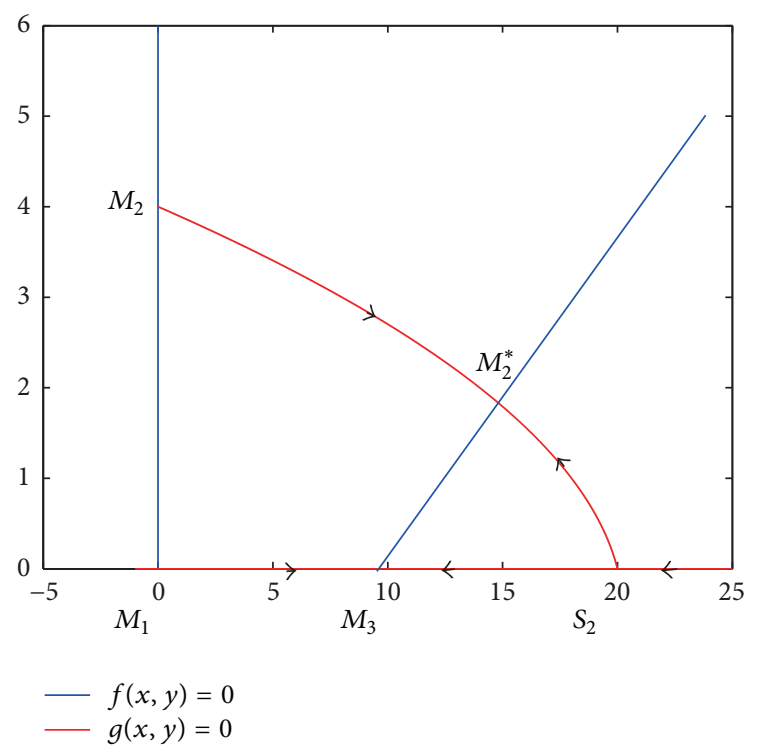

FIgURE $15: l \leq n, a b<\ln$, and $a=3$.

population density is suddenly decreased and the aphid ecosystem finally stays in the state of the aphid population absence. When the density of the aphid population of the initial state is lower than $y_{S_{1}}$, with gradual increase of the parameter $a$, the density of the aphid population in the stable state of the aphid ecosystem is gradually increased until the catastrophe occurs.

(2) If the environmental carrying capacity of the natural enemy population is equal to $x_{S_{2}}$ or smaller than $x_{S_{2}}$, the following biological significance is got: when the density of the aphid population of the initial state is higher than $y_{S_{1}}$, with gradual increase of the parameter $a$, the aphid ecosystem state is changed from the final state with the high aphid population density to the state of the aphid population absence, then to the beneficial catastrophe occurs. When the density of the aphid population of the initial state is lower than $y_{S_{1}}$, with gradual increase of the parameter $a$, the aphid ecosystem state is changed from the final state with the aphid population absence to the occurrence of catastrophe.

For the second category, with gradual increase of the parameter $a$, the aphid ecosystem state is changed from the state of the aphid population absence to the state with the high aphid population density.

According to the qualitative analysis and the biological significance above, the following two important results are got:

(1) If the point $M_{2}^{*}$ appears above the point $S_{1}$, then it must be a stable node. In this case, the solution to any initial state above the point $S_{1}$ will be stable at the point $M_{2}^{*}$, which represents the high density of the aphid population. This phenomenon is not what people want and the control is needed to reduce the density of the aphid population.

(2) For model (9), the curve segment $S_{1} S_{2}$ of the catastrophe manifold is in the refuge level of the aphid ecosystem, and once the system reaches the curve segment $S_{1} S_{2}$, it either becomes stable at the point $M_{3}$ or $M_{1}^{*}$ (that is, the aphid 


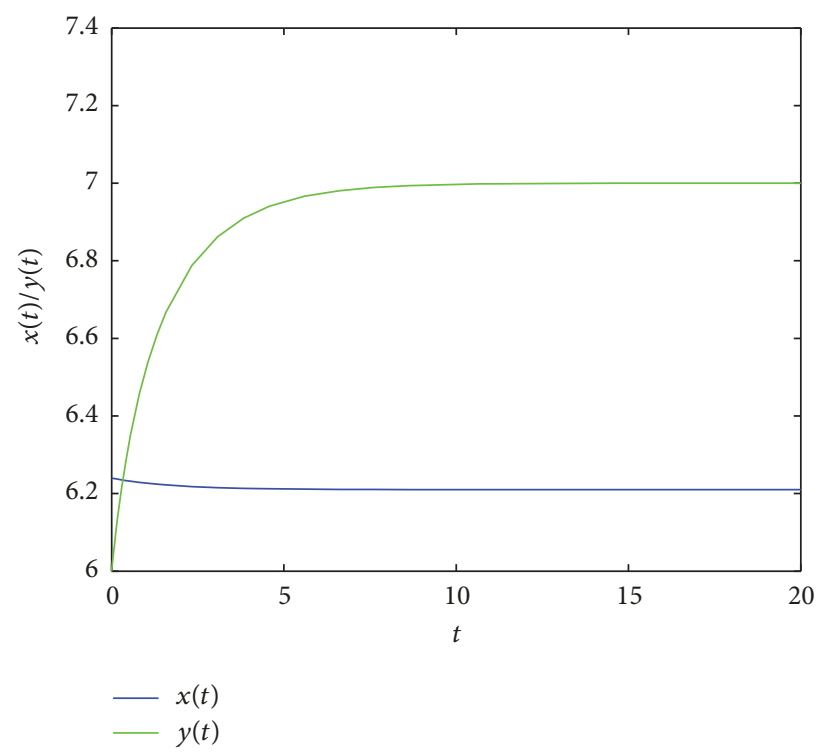

Figure 16: Time response before control.

systems stabilize at the low density of the aphid population) or causes a beneficial catastrophe to the $x$-axis after reaching the impasse point $S_{1}$.

The controllers are designed to manage the aphid ecosystems in the next section.

\section{Controller Design}

Taking releasing natural enemy as the control means, the corresponding control model of model (9) is proposed,

$$
\begin{aligned}
\frac{d x(\tau)}{d \tau} & =x(\tau)\left(a-\frac{x(\tau)}{y(\tau)+b}\right)+u(\tau), \\
0 & =y\left(l-y(\tau)-\frac{x(\tau)}{y(\tau)+n}\right),
\end{aligned}
$$

where $u(\tau)$ is the releasing amount of the natural enemy population and $u(\tau) \geq 0$.

Controller 11. The controller $u(\tau)$ is only related to the density of the natural enemy population, then $u(\tau)=F_{1}(x(\tau))$.

Controller 12. The controller $u(\tau)$ is related to both the density of the natural enemy population and the density of the aphid population, then $u(\tau)=F_{2}(x(\tau), y(\tau))$.

Without loss of generality, for Controller 11, take

$$
F_{1}(x(\tau))=c_{1} x(\tau), \quad c_{1}>0,
$$

and for Controller 12, take

$$
F_{2}(x(\tau), y(\tau))=x(\tau)\left(c_{2}-\frac{c_{3} x(\tau)}{y(\tau)+b}\right),
$$

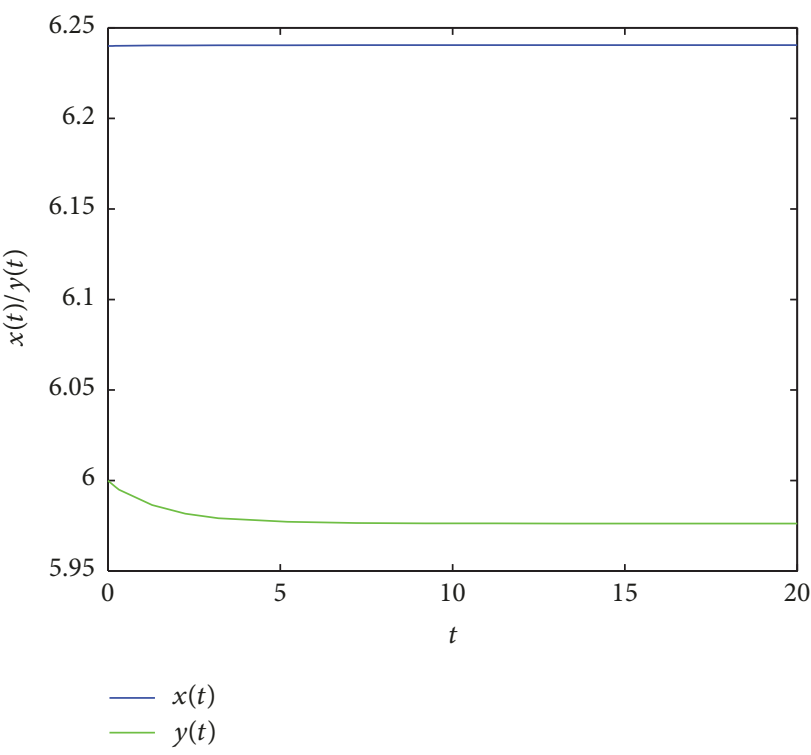

FIGURE 17: Time response after Controller 11 (stability).

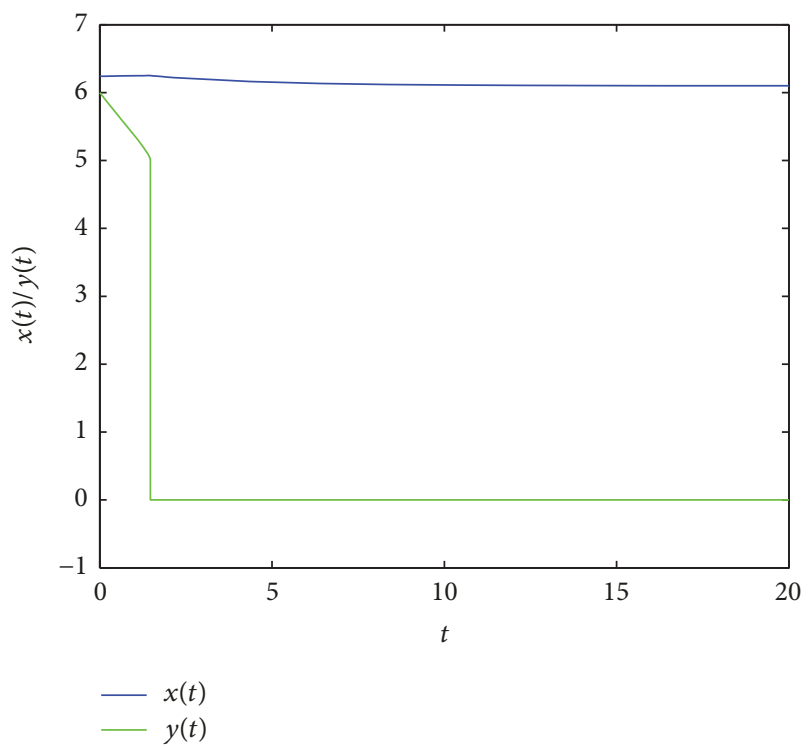

Figure 18: Time response after Controller 11 (catastrophe).

then the point $M_{2}^{*}$ does not appear above the point $S_{1}$ and either the aphid ecosystem stabilizes in the low density of the aphid population or a beneficial catastrophe occurs (see Figures 17-20).

Figure 16 shows the phenomenon that the system stabilizes in high density of the aphid population before control. For the phenomenon, Figures 17 and 19 verify Controllers 11 and 12 can make the system stable in low density of the aphids populations. Figures 18 and 20 show the catastrophe after control implementation, namely, the extinction of the aphids population, which is not ideal from the perspective of ecological diversity. So aiming at this situation, the controller parameters should continue to be adjusted to make the system stable in the low density of aphids population. Because 


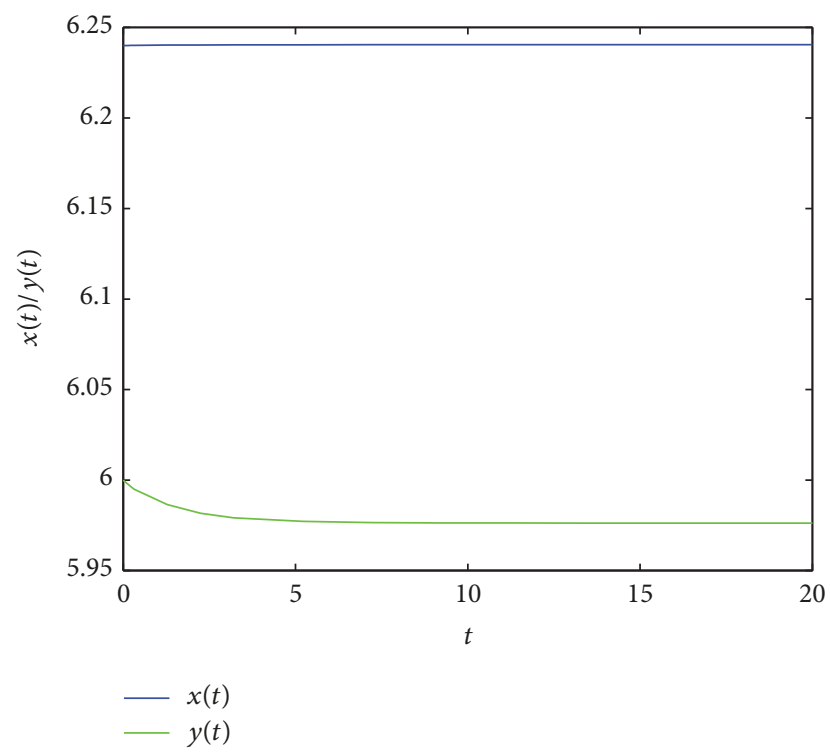

FIgURE 19: Time response after Controller 12 (stability).

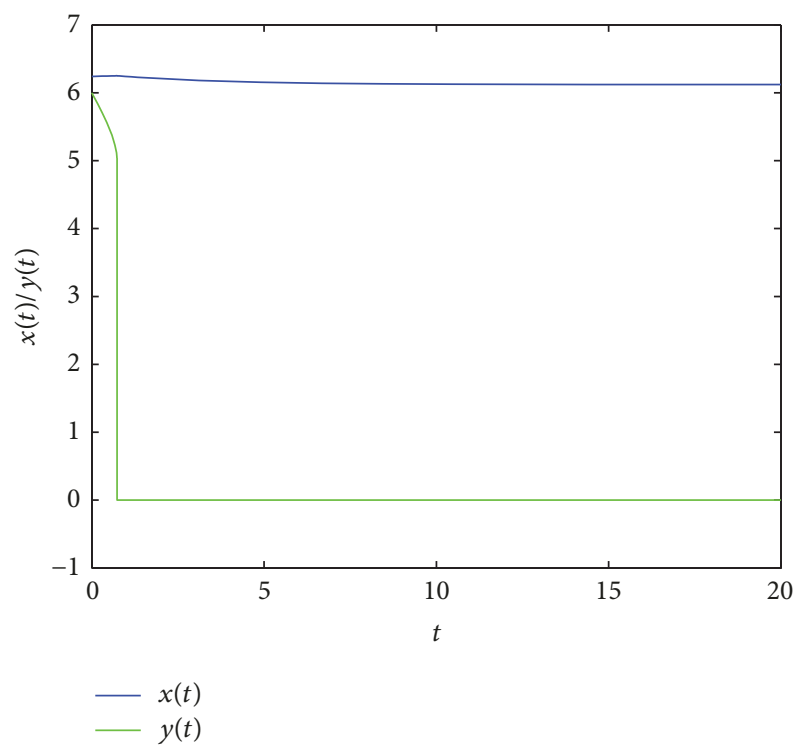

FIgURE 20: Time response after Controller 12 (catastrophe).

the controlled quantity can be determined by monitoring the density of the natural enemy population in the aphid ecosystem, it is easy to implement Controller 11. Although it is relatively complex to implement Controller 12 , it can effectively avoid using too many natural enemies in the state with low density of natural enemy population and density of the aphid population, so as to avoid unessential economic input.

\section{Conflicts of Interest}

The authors declare that they have no conflicts of interest.

\section{Acknowledgments}

This work is supported by the National Natural Science Foundation of China (11371081 and 11371030).

\section{References}

[1] S. Chander, L. R. Ahuja, F. B. Peairs, P. K. Aggarwal, and N. Kalra, "Modelling the effect of Russian wheat aphid Diuraphis noxia (Mordvilko) and weeds in winter wheat as guide to management," Agricultural Systems, vol. 88, pp. 494-513, 2006.

[2] M. K. D. K. Piyaratne, H. Y. Zhao, and Q. X. Meng, "Catastrophic behavior of aphid population dynamics: An analysis of swallowtail model," Computational Ecology and Software, vol. 5, pp. 775-788, 2014.

[3] D. Morgan, "Population dynamics of the bird cherry-oat aphid, Rhopalosiphum padi (L.), during the autumn and winter: A modelling approach," Agricultural and Forest Entomology, vol. 2, no. 4, pp. 297-304, 2000.

[4] H. R. Parry, A. J. Evans, and D. Morgan, "Aphid population response to agricultural landscape change: A spatially explicit, individual-based model," Ecological Modelling, vol. 199, no. 4, pp. 451-463, 2006.

[5] M. K. D. K. Piyaratne, H. Zhao, and Q. Meng, "APHIDSim: A population dynamics model for wheat aphids based on swallowtail catastrophe theory," Ecological Modelling, vol. 253, pp. 9-16, 2013.

[6] D. D. Jones, "Catastrophe theory applied to ecological systems," Simulation Transactions of the Society for Modeling and Simulation International, vol. 29, pp. 1-15, 1977.

[7] H. B. Wang and C. Jin, "Application of catastrophe theory in interspecies eology," Journal of Ecology, vol. 7, pp. 41-45, 1988.

[8] 1. R. Zhai, "Application of Spatial Analysis Technique in Pest Population Management," Journal of Applied Ecology, vol. 5, pp. 314-318, 1997.

[9] H. Y. Zhao and S. Z. Wang, "Apply catastrophe theory to study control strategy of aphid ecosystem," Chinese Science Bulletin, vol. 3422, pp. 1445-1949, 1989.

[10] X. D. Zhao, L. G. Zhu, H. Y. Zhao, and L. F. Zheng, "Prey under nature enemy model parameter grey estimattion," Journal of Northwest A F University (NatSciEd), vol. 36, pp. 185-188, 2008.

[11] D. M. Li, Z. Zhang, Z. F. Ma, and B. Y. Xie, "Allee effect and a catastrophe model of population dynamics," Discrete and Continuous Dynamical Systems-Series B, vol. 4, pp. 629-634, 2012.

[12] M. Yue and R. Schlueter, "Bifurcation subsystem and its application in power system analysis," IEEE Transactions on Power System, vol. 19, no. 4, pp. 1885-1893, 2004.

[13] S. Ayasun, C. O. Nwankpa, and H. G. Kwatny, "Computation of singular and singularity induced bifurcation points of differential-algebraic power system model," IEEE Transactions on Circuits System, vol. 5, pp. 1525-1537, 2004.

[14] W. Marszalek and Z. W. Trzaska, "Singularity-induced bifurcations in electrical power system," IEEE Transaction on Power System, vol. 20, pp. 302-310, 2005.

[15] I. O. Chua and A. Deng, "Impasse points. part I: numerrcal aspects," International Journal of Circuit Theory and Applications, vol. 17, pp. 213-225, 1989.

[16] I. O. Chua and A. Deng, "Impasse points. part II: numerrcal aspects," International Journal of Circuit Theory and Applications, vol. 17, pp. 271-282, 1989. 
[17] L. Dai, Singular Control System, Springer, New York, NY, USA, 1989. 


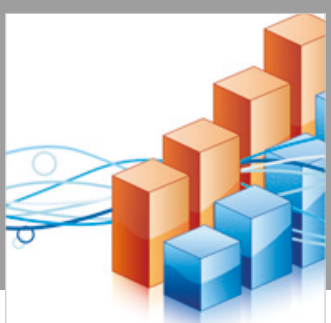

Advances in

Operations Research

\section{-n-m}
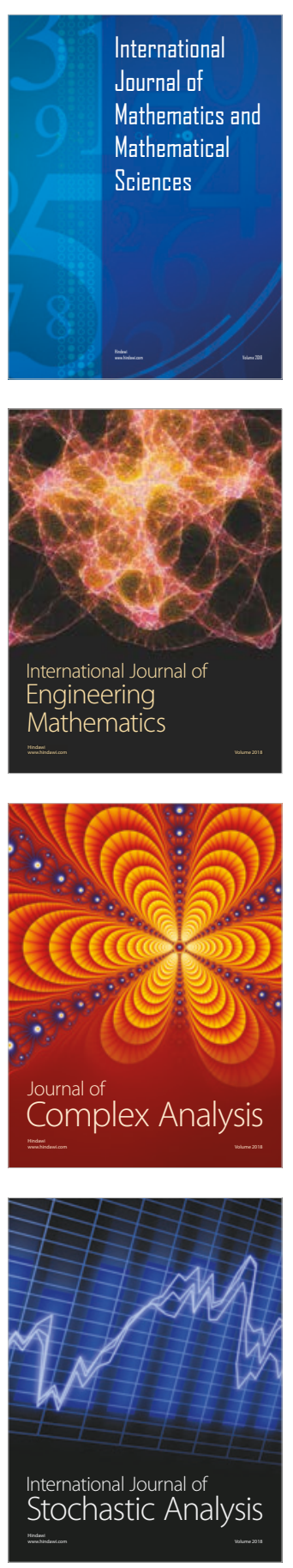
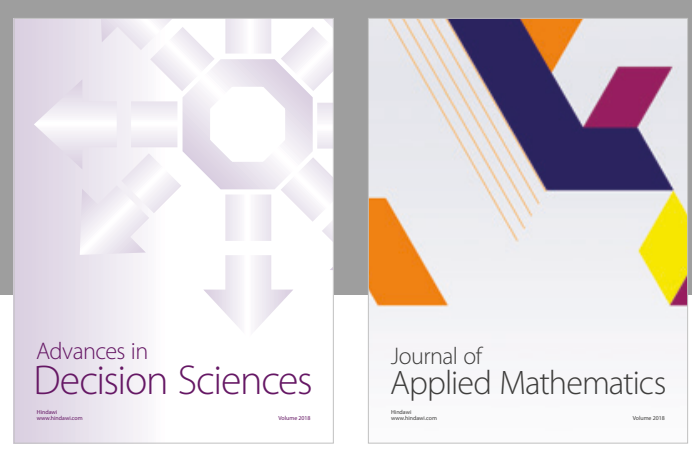

Journal of

Applied Mathematics
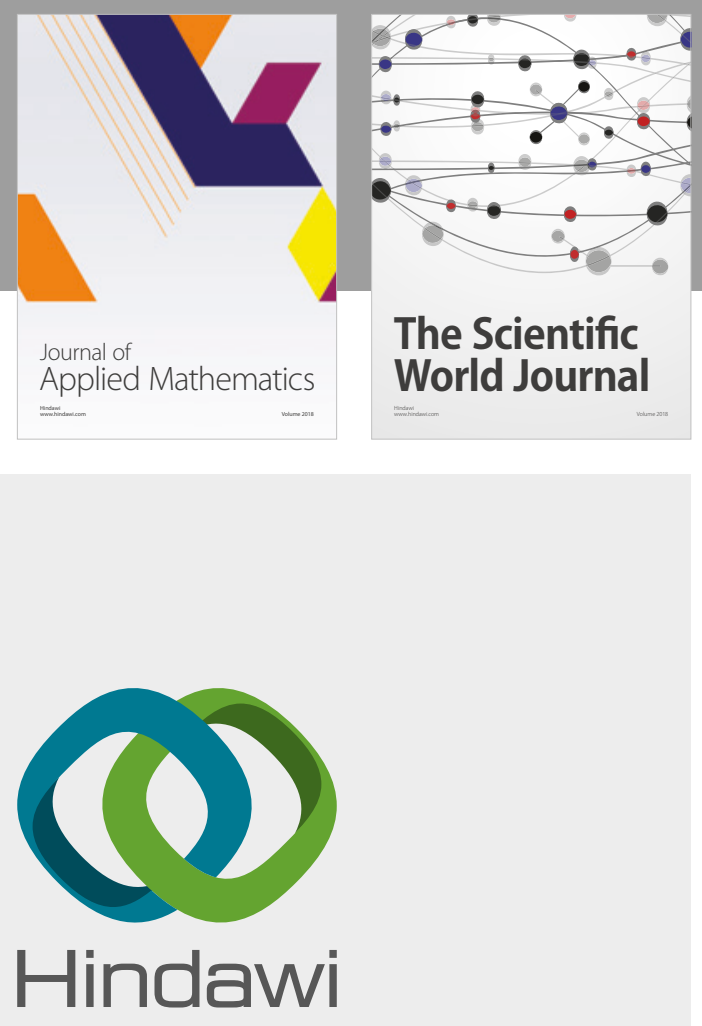

Submit your manuscripts at

www.hindawi.com

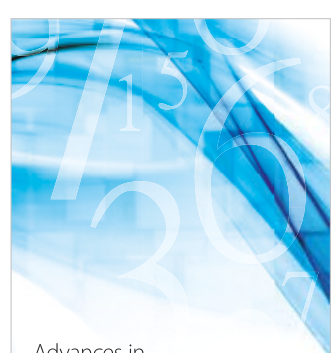

Advances in
Numerical Analysis
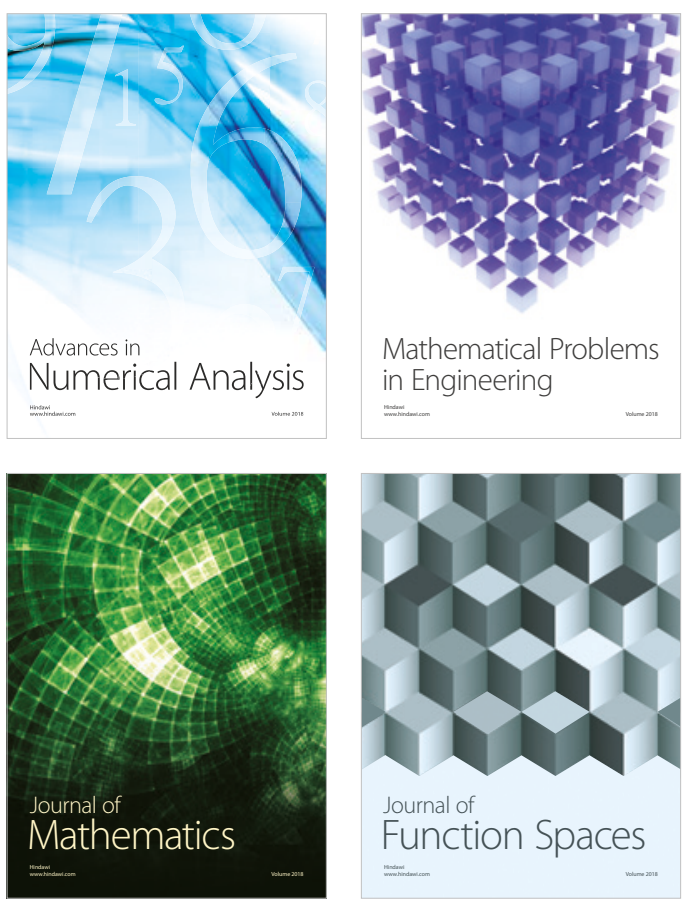

Mathematical Problems in Engineering

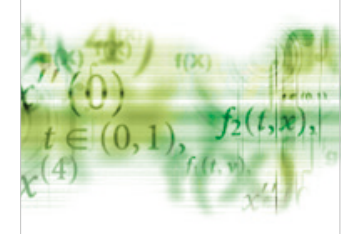

International Journal of

Differential Equations

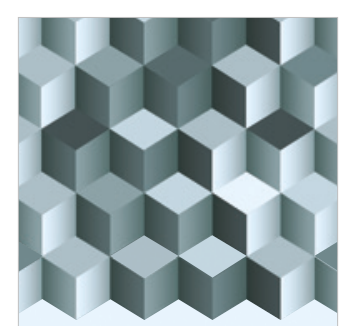

Journal of

Function Spaces

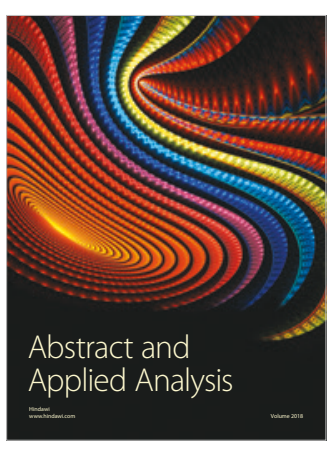

The Scientific

World Journal

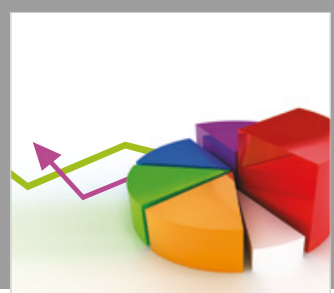

Journal of

Probability and Statistics
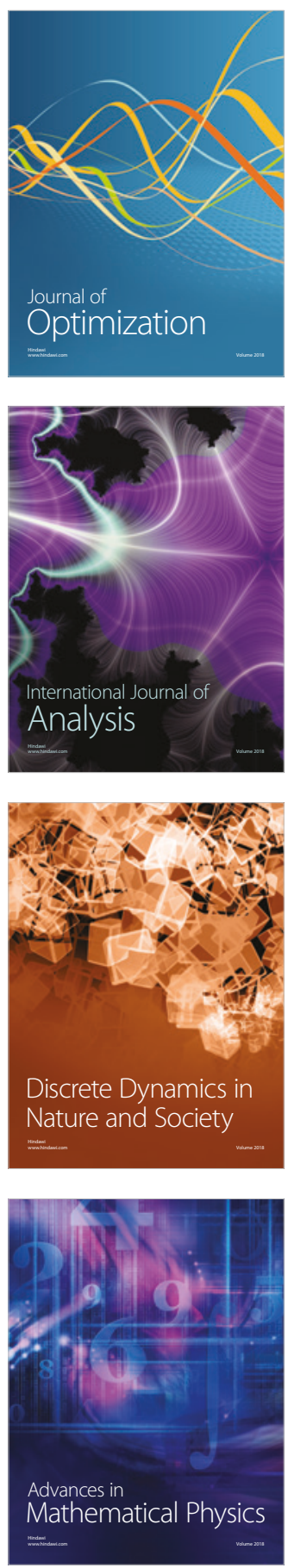